\title{
AC 2011-1638: A VEHICLE DYNAMICS DESIGN AND SIMULATION TOOL FOR CAPSTONE PROJECTS
}

\section{John E. Pakkala, Milwaukee School of Engineering}

John E. Pakkala is an Associate Professor of Mechanical Engineering at Milwaukee School of Engineering (MSOE). Before coming to MSOE, he spent more than twenty years as a special machine designer and was involved with the design, construction and installation of machines and manufacturing automation equipment for automotive, aerospace, and defense industry clients. Dr. Pakkala earned a Bachelor of Science degree in mechanical engineering from Michigan State University. His Master of Science and $\mathrm{Ph}$.D. degrees in electrical engineering from Michigan Technological University were in the area of dynamic control theory and system identification. He has published papers in fluid mechanics, internal combustion engine controls, system identification, and engineering education. He consults in the areas of manufacturing engineering and in vehicle crash reconstruction. He is currently the director of an exchange program for mechanical engineering students between MSOE and the Fachhochschule Lbeck in Lbeck, Germany. 


\section{A Vehicle Dynamics Design and Simulation Tool for Capstone Projects}

Abstract

A vehicle dynamics simulation tool developed by students and for use by student design teams is presented in this work. The project is the result of work done by students participating in an exchange program between international partner universities. Students in the exchange program complete a Senior Capstone Design project and additionally write a Diploma Thesis as part of earning degrees from both universities. The simulation tool is meant for use in the early stages of the design of four-wheeled vehicle projects such as the SAE Mini-Baja challenge or the SAE Formula competition. The simulation tool uses MATLAB and Simulink and simulates a14degrees-of-freedom (DOF) system. The model can accommodate different suspension linkages and allows anti-roll bars in the simulation and includes is capable of simulating Ackermann, parallel steering and reverse Ackermann steering. Non-linear tire modeling simulates turning forces from different types of tires on various surfaces. Results of low speed cornering simulation are verified by a physical test with a passenger vehicle at low velocities. The results of the high velocity cornering analysis are cross-referenced with findings in vehicle dynamics literature. Simulations provide turning radius estimation and the determination of the under-steer and over-steer behavior of the vehicle. Suspension impact forces and suspension travel in cases of impact from a user specified height are included. The design and simulation tool is opensource allowing future teams access to the software for future updates or revisions. A tutorial is included that provides teams with instructions on the software usage, facilitating the decisionmaking process earlier in the capstone design schedule than would otherwise be possible.

\section{Background}

At the Milwaukee School of Engineering (MSOE) Mechanical Engineering students are required to complete either a two-term or three-term Senior Design sequence. Most students opt to complete the three-course sequence that begins in September and ends in May. The first term finds students developing a proposal for their group's design goals for the year. In the second term analysis, design and initial building or testing is accomplished. The third term in spring is devoted to building the project and perhaps bringing a device to a competition such as the SAE Mini-Baja, or SAE Aero competition, among others.

\section{Foreign Exchange Program and the Diploma Thesis}

MSOE has maintained an exchange program with the Lübeck University of Applied Sciences, or Fachhochschule Lübeck (FHL), for the past fifteen years beginning in the respective universities' electrical engineering departments. The mechanical engineering exchange program has been in operation for seven years. Approximately twelve mechanical engineering students from MSOE spend their entire third year studying in Lübeck, Germany alongside approximately twelve of their FHL exchange program counterparts. Then both MSOE and FHL students spend their fourth year in the USA. Completion of the program results in earning degrees from both universities. 
For program participants, the MSOE degree requires completion of the three-term senior design sequence. In addition, the FHL degree requires the completion of a Diploma Thesis. The Diploma Thesis is expected to be a rigorous formal technical report. Students are advised that a Diploma Thesis is a "statement that justifies earning a diploma". In that sense, students are expected to define an engineering problem to solve, systematically develop the best solution to the problem, then implement and test the solution.

Senior design projects and thesis works may be done in parallel. However, the senior design projects are normally thought of as team efforts, while the thesis works must be produced by each student individually. Consequently, most program participants make efforts to use portions or all of their contributions to the team effort as work that will ultimately form their thesis. Of course, this may not be entirely possible as group goals may at times conflict with personal thesis goals. This conflict of goals is a fair representation what is often found in professional engineering circumstances and is a good experience for undergraduate students.

\section{Vehicle Dynamics and Vehicle Design}

As is the case with many undergraduate capstone programs, some projects, such as the SAE Mini-Baja vehicle design competition, are repeated year after year. But the incoming senior students may have little or no experience with any of the previous years' vehicles or with the previous students. Information is relayed in the form of technical reports, or by a faculty advisor who might also provide new goals each year for design or performance improvements.

Vehicle dynamics is a complicated topic, especially for undergraduates. At Milwaukee School of Engineering, students may elect to take a course in introductory vehicle dynamics, but it is limited in scope to an introductory level. Thus students have a difficult time calculating, e.g., a turning radius at various speeds due to the many factors involved such as tire dynamics, suspension effects, and tire-to-road interface functions. Consequently, it is often the case that students may be aware that, for instance, the previous year's vehicle had poor handling characteristics in a certain competition event. Based on this information they proceed to make alterations to the vehicle to improve, say, the steering capabilities. This may be accomplished by shortening the wheelbase or redesigning the steering linkages. However, it is not always clear what effect their changes will have until the vehicle is built and tested. One goal of the tool in this work is to offer students a better chance at predicting the new performance levels by conducting and comparing simulations resulting from several design change schemes.

Vehicle dynamics software packages are available on the market. At MSOE as is true on many campuses, students have access to one or several vehicle dynamics software programs that possess the capability to simulate vehicle dynamics under various operating conditions. These software packages allow students to vary vehicle design parameters and then simulate the dynamics ultimately facilitating a design decision. Unfortunately from a pedagogical standpoint, much of the software is a 'black box' and students gain only limited experience in the underlying equations of motion necessary to effect simulations. Also, the software usually has good graphics capabilities and students tend to spend a great deal of time producing interesting graphical presentations, but at the expense of developing the equations of motion. 
Now on the other hand, given the time constraints placed on undergraduates in their final year coupled with the task of actually building a vehicle, it may be a bit unrealistic to expect them to also develop multi-degree-of-freedom equations and write a numerical solver routine that accounts for the linear and nonlinear components of a vehicle. Consequently, analyses completed by students are usually limited to single-degree-of-freedom analyses such as the bounce and jounce of a quarter-car model that remains in contact with the ground.

It is not the intention to suggest that 'black box' simulations are necessarily inappropriate tools for learning. The purpose of this paper is to show an alternative approach used at MSOE that is meant to strike a balance between working from basic principles and using existing modeling software.

\section{Student Projects}

As mentioned earlier in this paper, MSOE and FHL students participating in the exchange program must write a Diploma Thesis to earn the FHL degree. Two of these students chose to write theses on vehicle dynamics and to also develop MATLAB ${ }^{\circledR}$ and Simulink $₫$ based solvers to simulate vehicle dynamics. Part of the motivation for the development of these two theses was to provide an early design tool for future vehicle design teams. The solvers may be thought of as more like 'gray boxes' in the sense that students need to read and understand the code as written, including examining the equations of motion and their multi-degree of freedom couplings. In addition, nonlinear functions such as tire lateral forces may be altered within the solver routines to make design decisions for vehicle components. Much of the detail provided in this work is the result of those two theses. [1,2]

\section{Dynamics Modeling}

The modeling in the design tool is quite comprehensive and only a few of the modeling and simulation methods and results are provided in this paper. The modeling and solvers were designed to simulate the behavior of a vehicle under various inputs by both the road and the driver. Using these simulations, the influence of different design parameters on the vehicle behavior may be evaluated.

The vehicle is modeled as a 14-DOF system consisting of three translational movements of the vehicle in its three local axes and the three rotations around these axes. Additionally, each wheel is able to move in the vertical direction and can rotate around its axis. Forces and model parameters, such as time-varying tire loads are also calculated and simulated.

Aerodynamic forces are not taken into account in this design tool. One reason for this is that student competition vehicles' speeds are limited and the aerodynamic forces are assumed to be small [3]. Additionally, the aerodynamic forces vary greatly for possible shapes of the vehicle [3]. Of course, it is conceivable to add aerodynamics to the models, a fact that may influence future Diploma Thesis works! 
Students designing a specific vehicle will be concerned with many aspects of vehicle dynamics and performance goals. Handling is one significant aspect to consider. Handling performance is related the turning radius of the vehicle. Additionally, the brake-loose behavior of the tires is a parameter of interest. In this design tool, under-steering or over-steering behavior can be determined by examining the yaw rotation of the vehicle, the steering angle and the vehicle's velocity.

Another parameter of interest is the roll and rollover behavior of the vehicle due to lateral acceleration and road inputs. The vehicle roll is represented by the roll angle. The normal forces of the tires show under which situations the tires lose contact with the ground. With this information, a rollover risk can be determined.

The responses of the vehicle to road height changes are calculated in terms of acceleration, roll and pitch angles of the sprung mass. Additionally, the position of the vehicle's unsprung mass is significant in order to determine the suspension travel. The suspension travel and forces are also of interest in the case of impact after a jump of the vehicle. The response of the vehicle to vibrations is also a significant factor of the vehicle's dynamics, which may be represented by calculation of the natural frequencies and the damping ratios of the vehicle.

\section{Coordinate Systems}

To simulate the dynamics of the vehicle and to track the position of the vehicle a global and a local coordinate system are required. Both coordinate systems are derived using the appropriate transformation coupling equations. The global coordinate system is fixed to the ground and the local coordinate system is fixed to the vehicle. Both are right-hand coordinate systems, in which the z-axis is vertical and defined positive in the upward direction. The global coordinate system is used to track the movement of the vehicle in its surrounding world. The local coordinate system is fixed to the vehicle as shown in Figure 1. The origin of the coordinate system is at the center of gravity $(\mathrm{COG})$. The roll rotation is the rotation of the sprung mass around the roll axis, which is nearly parallel to the local $\mathrm{x}$-axis. The pitch rotation is around the pitch axis, which is parallel to the local y-axis. The yaw rotation is around the local z-axis. 


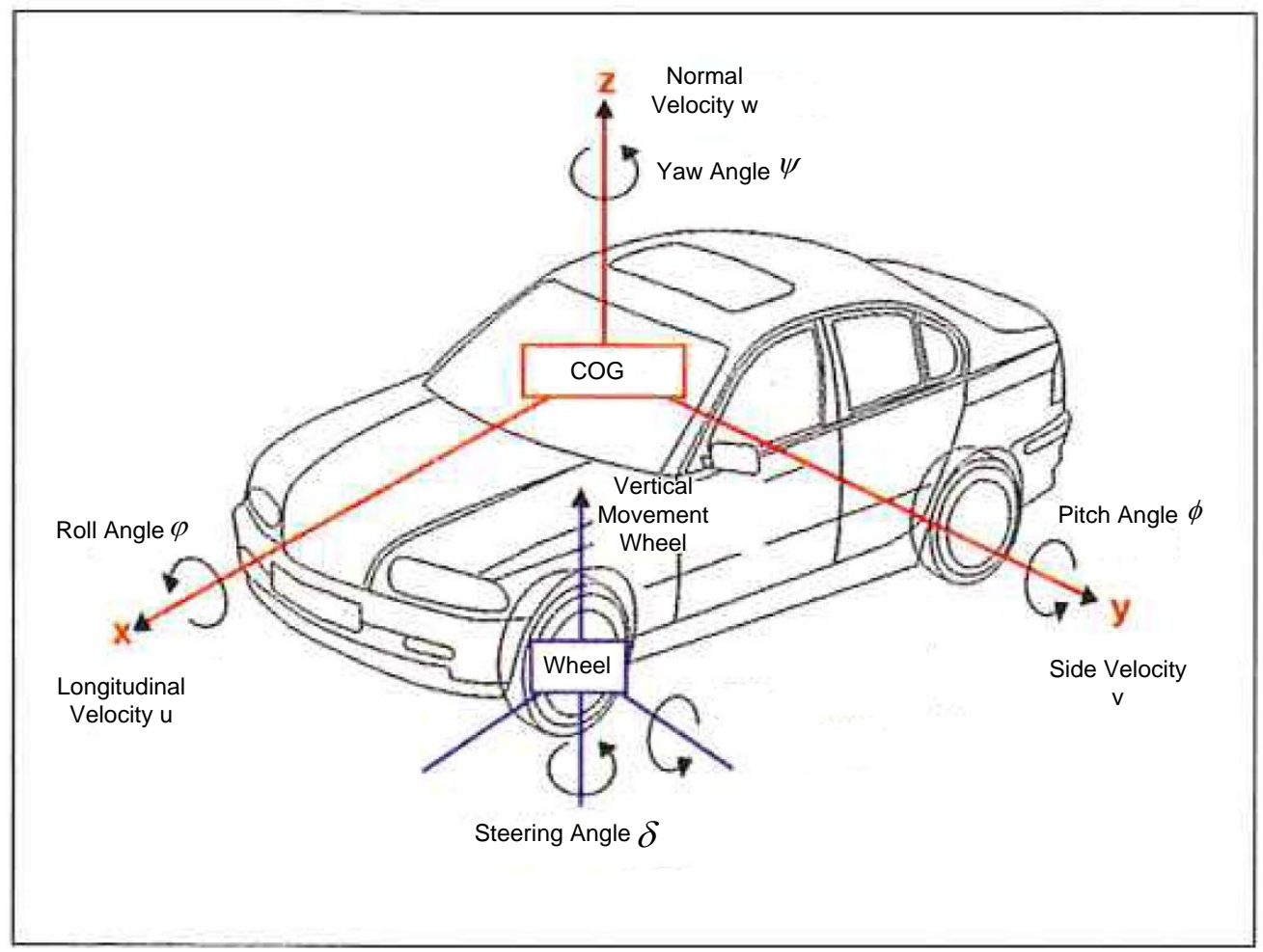

Figure 1: Vehicle coordinate system according to ISO 8855/DIN 70000[4]

For the transformation between the local and the global coordinate system, the assumption is made that all roll and pitch angles are small. Therefore, the influence of these angles can be neglected. Otherwise it would be necessary to consider the rotations' affect on each other. In that case the formulas for the Euler angles would be required to compute the orientation of the vehicle in a 3-dimensional space [5]. The Euler angles are a method to describe the orientation of a rigid body (coordinate system) by a sequence of three rotations. If two axes are rotated in a way that they are in the same space, one degree of freedom is lost. This phenomenon is called "Gimbal Lock" [6]. All angles except the yaw angle are relatively small; therefore this situation does not occur, although the rotational transformations could be the subject of a future Diploma Thesis. Figure 2 shows how the global and local coordinate systems are related to each other. The local coordinate system is rotated by the yaw angle $\psi$ to the global coordinate system and has a displacement of $x_{C O G G}, y_{C O G G}$ to its origin. 


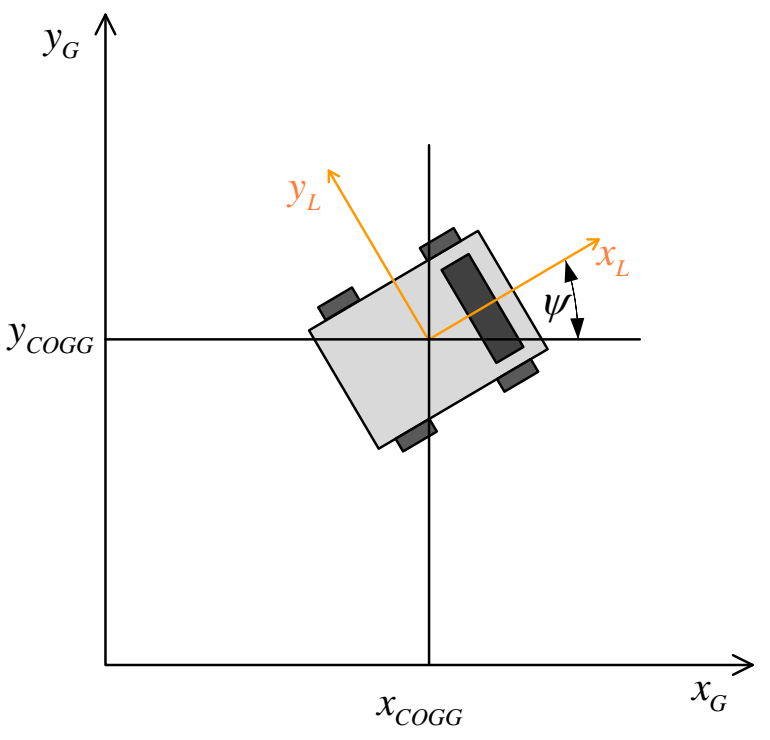

Figure 2: Global and local coordinate system [1]

The transformation between the local and the global coordinate system requires the local velocities $v_{x}, v_{y}$ and the yaw angle $\psi$.

$x_{C O G G}=x_{C O G G}+\Delta x_{C O G G}$
$y_{C O G G}=y_{C O G G}+\Delta y_{C O G G}$

The equations may be derived from Figure 2 and using the trigonometric functions

$$
\begin{aligned}
& \Delta \dot{x}_{C O G G}=\cos (\psi) v_{x}-\sin (\psi) v_{y} \\
& \Delta \dot{y}_{C O G G}=\sin (\psi) v_{x}+\cos (\psi) v_{y}
\end{aligned}
$$

\section{Suspension Modeling}

A three-dimensional sketch of the system is shown in Figure 3. The vehicle's body is connected to the four wheels by the four suspension systems. The front of the vehicle is on the right side of the sketch, in the positive $\mathrm{x}$-direction. Springs and dampers are modeled for all four shock absorbers and all four tires. All differential equations are reduced to first-order and set into state model form for use in Simulink modeling. 


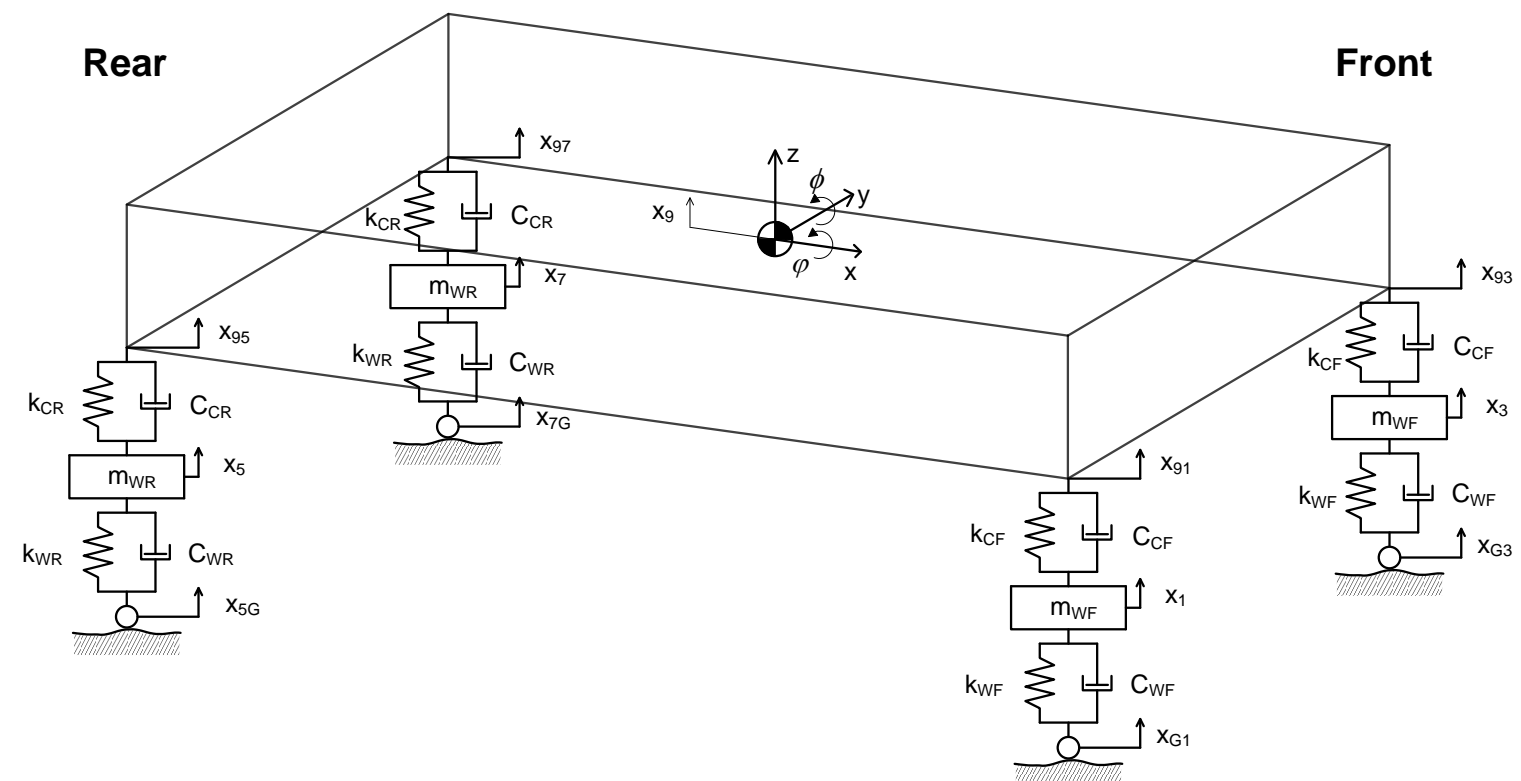

Figure 3. Sketch of a 7 DOF model [1].

Figure shows the side view of the vehicle's body with its COG and the pitch center. The points $x_{95 / 7}$ are the left and right connecting points to the rear suspension. The points $x_{91 / 3}$ are the left and right connecting points to the front suspension.

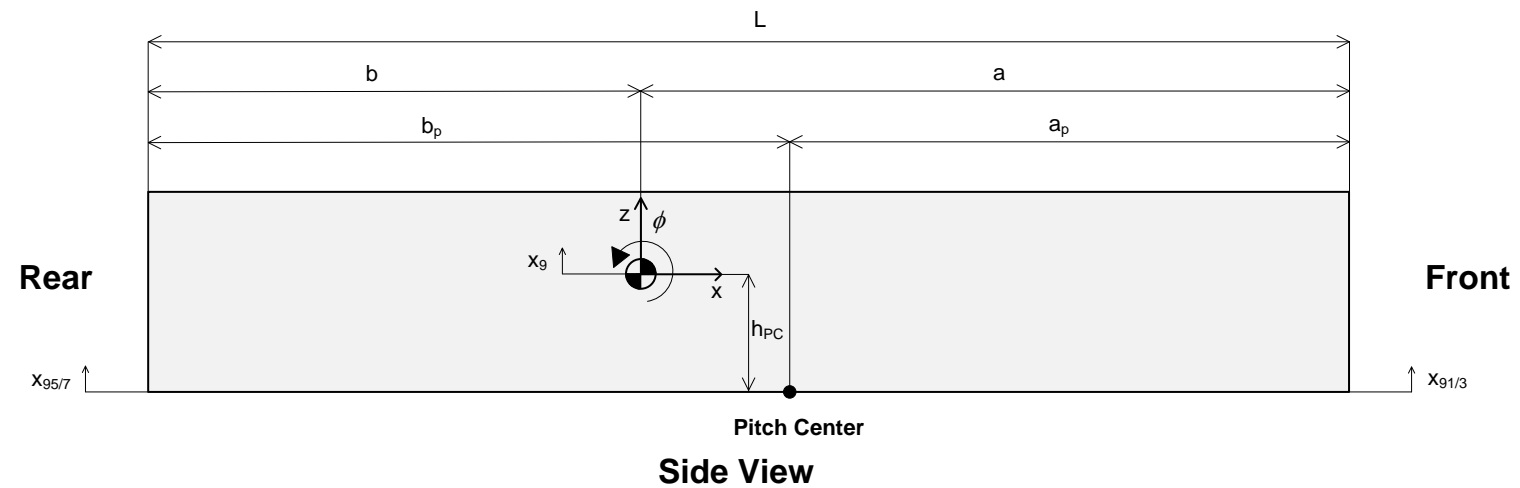

Figure 4. Side view of the 7-DOF model [1]

The front view of the vehicle's body is shown in Figure 5. The points $x_{91 / 5}$ are the connection points to the suspension system on the right side of the vehicle. The points $x_{93 / 7}$ are the ones on the left side of the vehicle. 


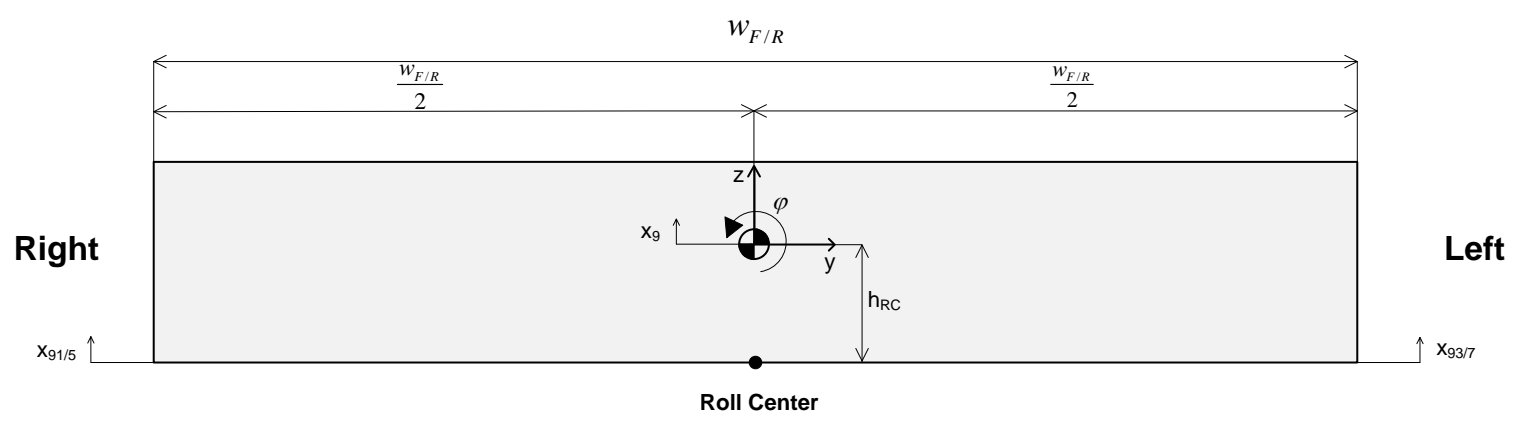

Front View

Figure 5. Front view of the 7-DOF model [1]

The simulation tool takes note of the change in load distribution caused by roll or pitch when the roll/pitch axis does not intercept the COG. The situation is depicted in the front view of the simulated vehicle in Figure 6 for the rotation around the roll axis.

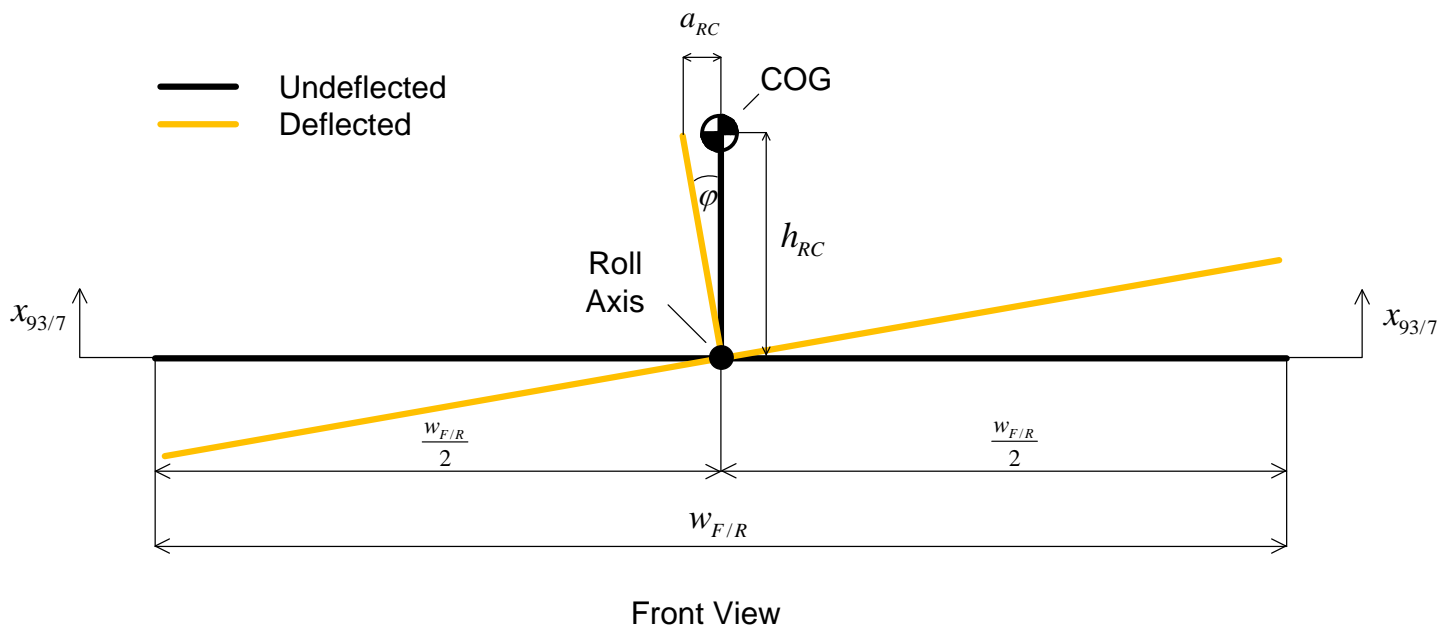

Figure 6. Load distribution by a roll angle [1]

The horizontal displacement, for relatively small angles, of the COG is given by

$a_{R C}=h_{R C} \cdot \sin (\varphi) \approx h_{R C} \cdot \varphi$.

Therefore, the resulting moment around the roll center is

$M_{L D R}=a_{R C} \cdot m_{c a r} \cdot g \approx h_{R C} \cdot m_{c a r} \cdot g \cdot \varphi$.

The same derivation can be applied to calculate the load distribution caused by pitch motion resulting in

$M_{L D P} \approx h_{P C} \cdot m_{\text {car }} \cdot g \cdot \phi$. 
Simulation and Results

All time-dependent functions are implemented in the SIMULINK block diagram, but only the main level is shown in Figure 7. It consists of a subsystem which generates the road zcoordinates called "Road", a subsystem which generates the driver inputs called "Driver", and a subsystem covering the dynamics of the vehicle called "Vehicle".

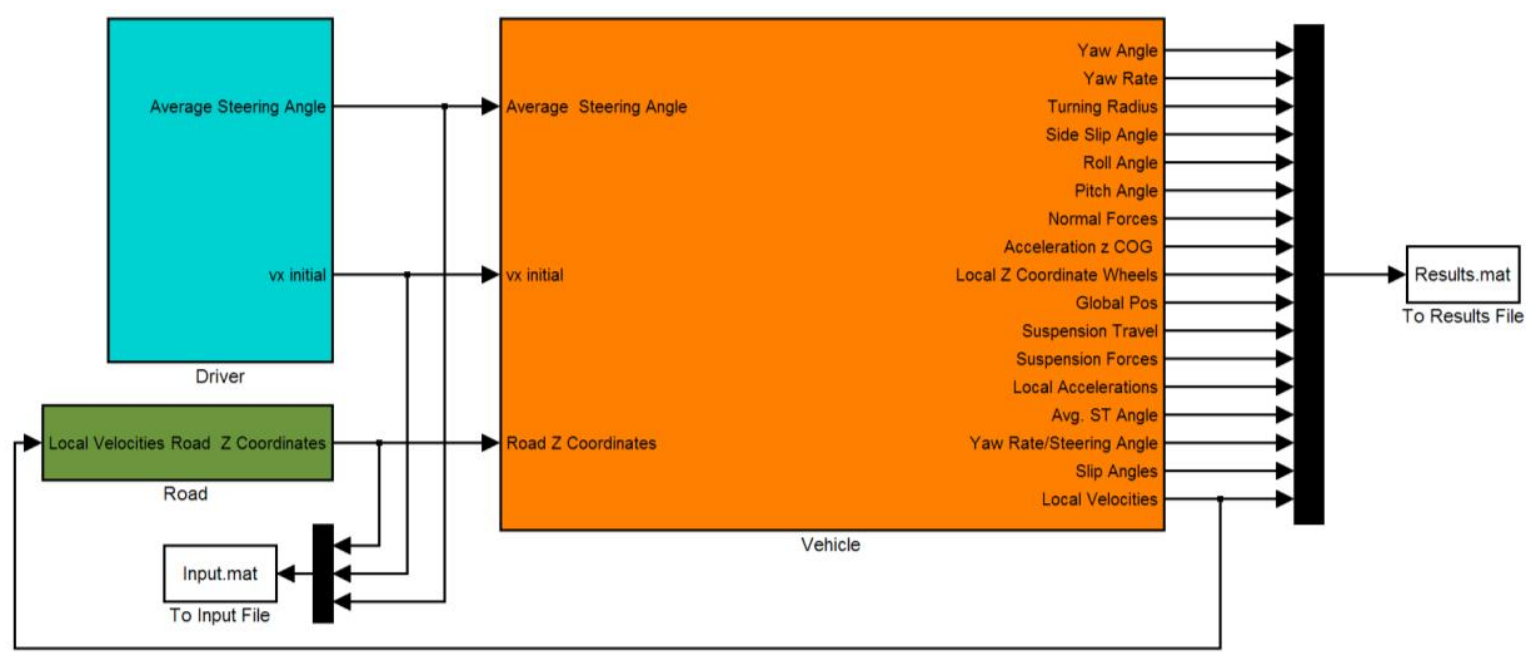

Figure 7. Main level SIMULINK block diagram [1].

In Figure 7 it is seen that the driver subsystem consists of different inputs, each of which can be a step or a ramp input, or any other function, and are controlled from a master m-file.

Several deeper levels of subsystems and sub-subsystems are used in order to compute the various inputs and outputs used in the main level, e.g., the tires' lateral forces are calculated using nonlinear functions matched to each tire and depend on the several forces acting on each tire at any time instant. See Figure 8 taken from [2]. Any of the inputs and outputs may be stored in data files in order to plot and analyze the simulation results.

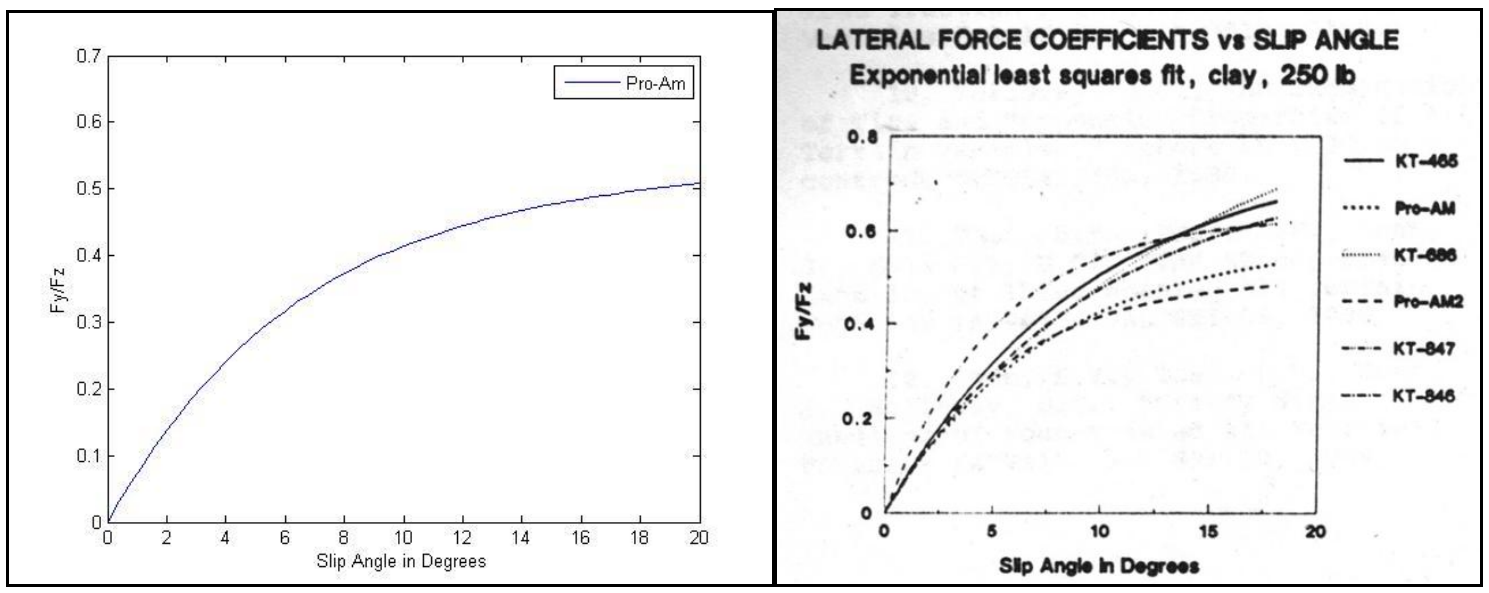

Figure 8 Lateral forces on tires, calculated (left) [2], and in literature (right) [7]. 
A typical simulation might be the calculation of the response of the vehicle to a vertical step input of $0.1 \mathrm{~m}$ to all wheels at the same time. The response of the wheels and the COG is shown in Figure 9.
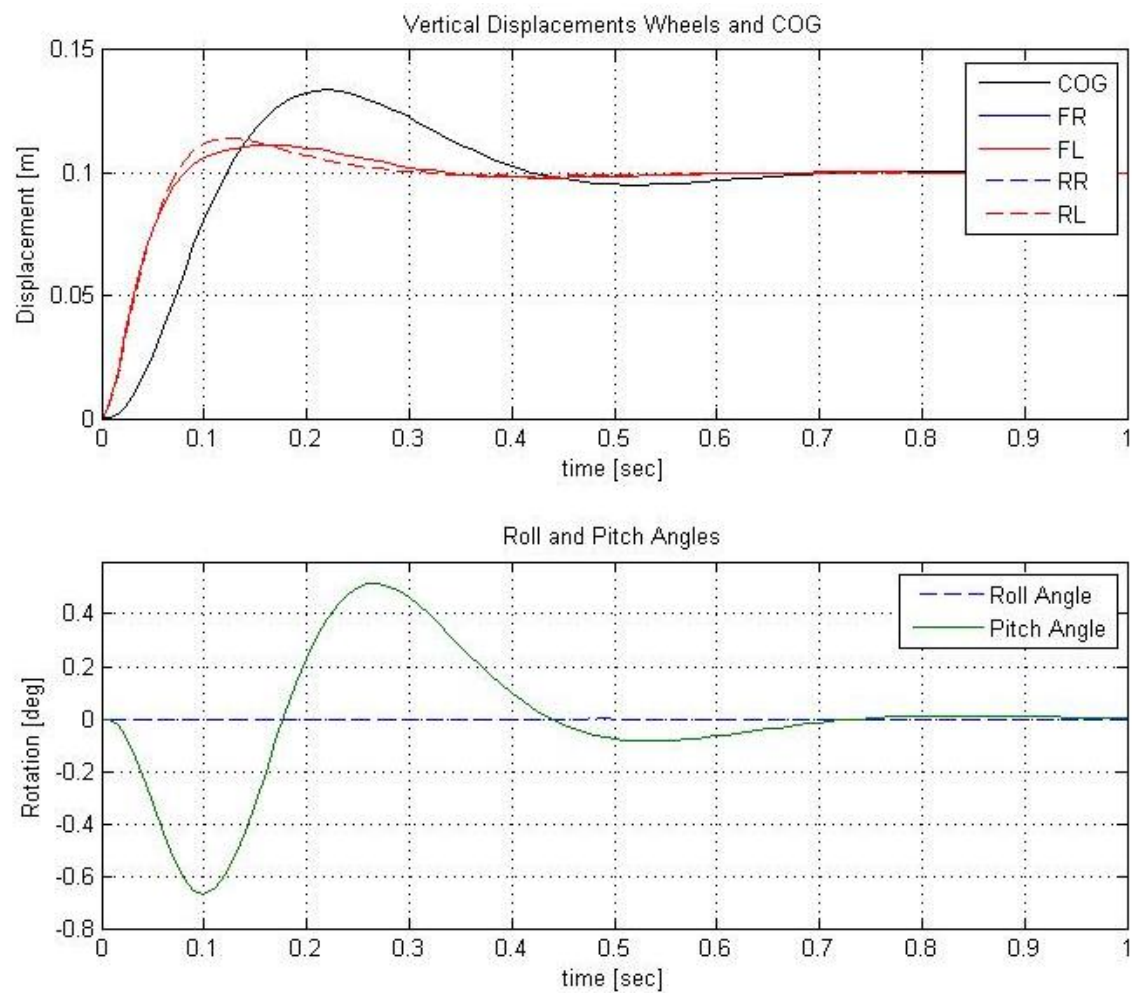

Figure 9. Response to step input at all four wheels.

The results in Figure 9 show that the left and right tires follow the same curve, as expected since they have the same suspension system, but front and rear are different due to different spring and damper selection. Similarly, the pitch angle changes are due to different front and rear shocks, but no roll occurs in this case.

Under true road conditions, step inputs do not occur because tires are round and would not hit the obstacle initially at its lowest point. Therefore, a step input of the road, which is smaller than the radius of the tire, would be more nearly a ramp input for the model. Additionally, the tire would deflect in the longitudinal direction when hitting a step, which causes a deceleration of the vehicle. Both phenomena also occur for ramp inputs, but are considerably smaller for small slopes. The tool can accommodate any input, but in this work ramp inputs are used to simulate road inputs. Longitudinal tire deflection phenomena are not taken into account in this design tool.

The slopes of the ramp inputs for the road height are dependent on the modeled bump and on the velocity of the vehicle. The bump is simulated with a delay time for the rear wheels, which is dependent on the wheelbase and the forward velocity of the vehicle. A combination of ramp inputs is used to simulate any desired road input.

To analyze the effect of any single system variable, the simulation may be performed for different values of this variable while keeping all other system variables constant. This results in 
a family of functions, in which the effect of the analyzed variable is visible. An example is shown in Figure 10 in which the suspension spring stiffness varied from in a range of $\pm 30 \%$ of the original value.
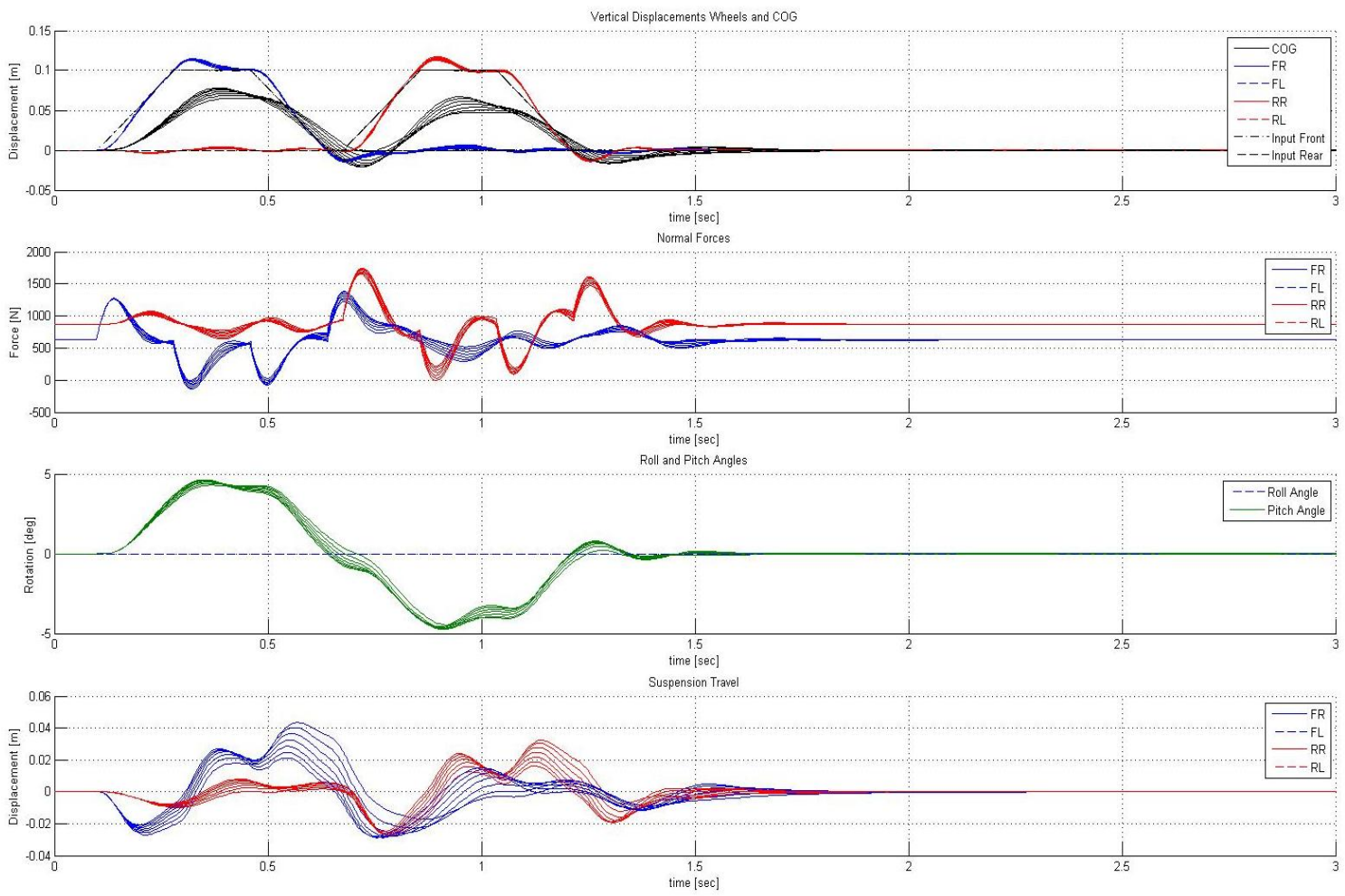

Figure 10. Vehicle responses shown in upper to lower subplots are: body and COG displacement, normal forces, roll and pitch angles, and suspension travels. The multiple plots reflect varying stiffness parameter values.

These data can be analyzed by comparing the peak values or standard deviations of the different graphs. It can be seen that the normal force peaks increase with increasing suspension stiffness and the suspension travel decreases. The analyses may be performed at different velocities to vary the road input frequencies to ensure correlation to generally accepted valid results.

The maximum suspension travel and the maximum forces may be determined for a simulated impact of the vehicle after a jump. This is executed by an initial condition for the velocity of the sprung and unsprung masses. This simulation starts at the equilibrium point for the vehicle load. In reality, the suspension would start with the wheels in the lowest possible position. Hence, the computed suspension travel and forces are slightly larger than the real ones. Figure 11 shows the suspension forces and the suspension travel for an impact of the vehicle from $0.5 \mathrm{~m}(1.64 \mathrm{ft})$. In this simulation, all four wheels hit the ground at the same time and the roll- and pitch angles are zero. The results for this particular simulation are a peak force for the front of $3 \mathrm{kN}$ and for the rear $4 \mathrm{kN}$. The suspension travel is for the front suspension $0.13 \mathrm{~m}$ and for the rear suspension $0.17 \mathrm{~m}$. The rear suspension travel is higher than in the front because the COG is located closer to the rear axle of the vehicle. 

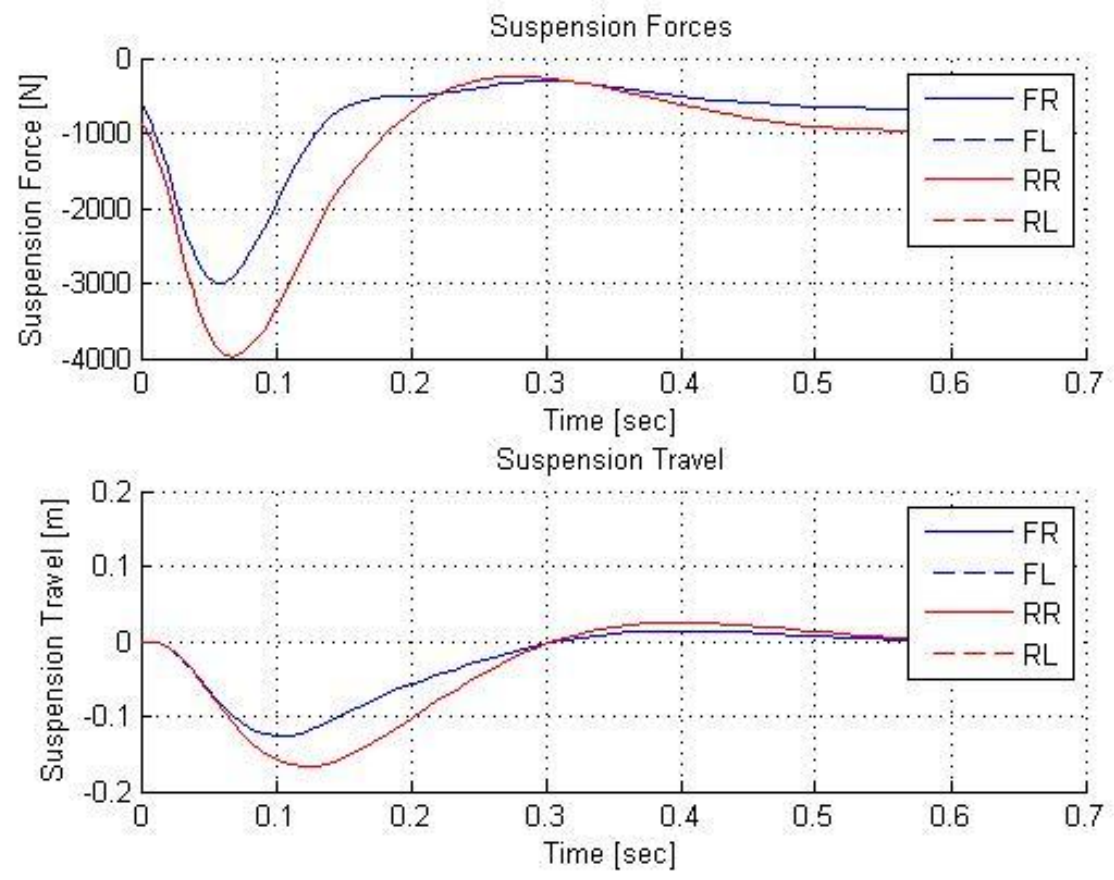

Figure 11. Simulation of the vehicles impact on the ground from $0.5 \mathrm{~m}$ height

The same simulation can be performed with one, two or three wheels hitting the ground before the other wheels hit the ground. This is realized by letting the vehicle fall on an uneven surface. Hence, the road $\mathrm{z}$-coordinate has the highest value for the wheel that hits the ground first. This simulation does not perfectly represent the real case, because the final steady-state values are slightly different compared to the case of a level road and a vehicle falling with a pitch and roll angle. However, the force peaks are assumed to be a good approximation to the force peaks.

The simulations presented here, while not entirely accurate or indicative of all possible scenarios, still provide enough information for students to make reasoned judgments about suspension component design and selection.

\section{Steady-State Cornering}

The simplest cornering simulation is steady-state cornering in which the vehicle drives at a constant velocity and a constant steering angle. The simulations can be performed using any steering input. Figure shows the steady-state cornering behavior of an under-steering vehicle on the left and of an over-steering vehicle on the right. Both are simulated using a step change from zero to fifteen degrees in the steering angle. It can be seen that in both plots of Figure 12 the turning radius increases with increasing velocities. This is due to centrifugal forces that increase with the square of the vehicle's longitudinal velocity. The responses of the under-steering vehicle are stable for all velocities and reach a steady-state value after about one second for the $45 \mathrm{~km} / \mathrm{h}$ simulation. The response of the over-steering vehicle is only constant for the simulated velocities below $25 \mathrm{~km} / \mathrm{h}$. The responses for the higher velocities show oscillatory behavior. This is interpreted as a spinout, or a loss of static friction on the tires. 
This loss of fidelity in the numerical simulations is one of the positive aspects of the 'gray box' nature of this design tool. Students must interpret the simulation results, especially where the methods fail, or when the results reveal the limits of the assumed modeling.
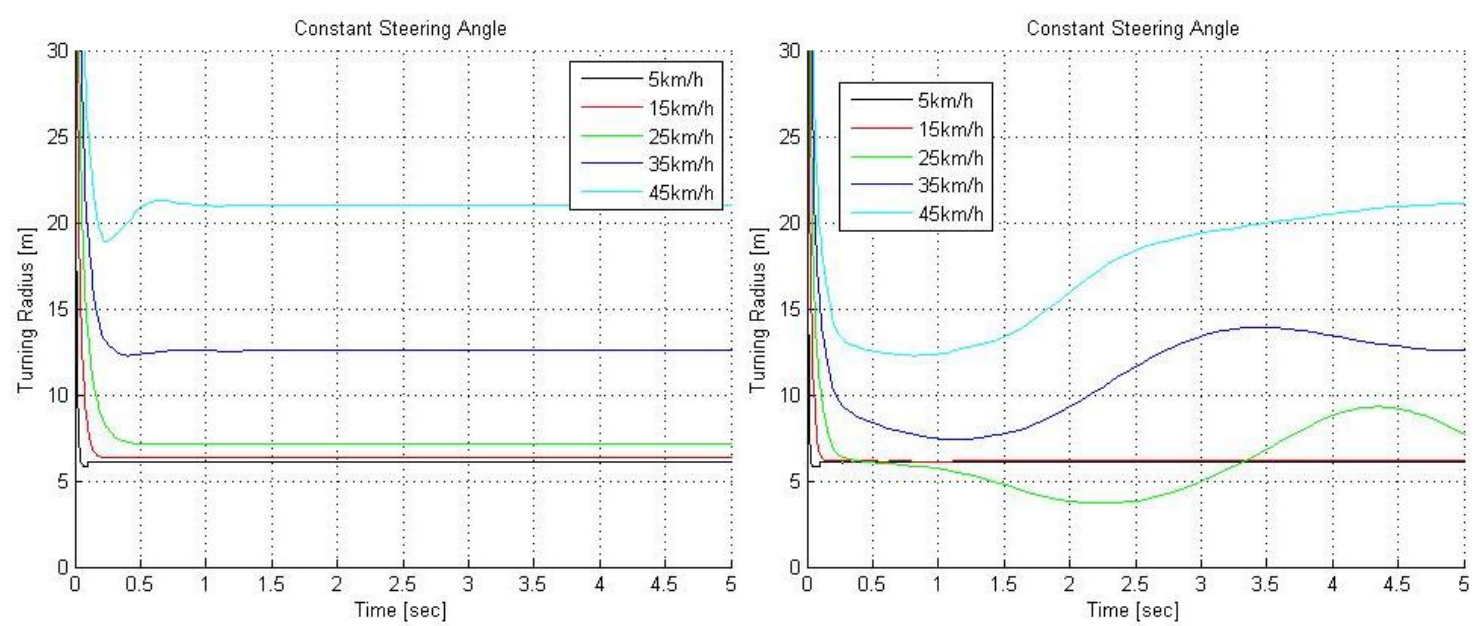

Figure 12. Steady-state cornering at fifteen degrees steering angle for an under- and oversteering vehicle

\section{Wheelbase}

The analysis of the effect of the wheelbase is performed with an under-steering and an oversteering vehicle, which differ only by their COG position. The distinction between these two vehicle types is examined because analyses show that changes in the wheelbase do not change the under-steering behavior in over-steering behavior.

Figure 13 shows the turning radius over the velocity and the yaw velocity gain for an understeering vehicle with different wheelbases at a steering angle of 15 degrees. The turning radius for velocities below $\sim 27 \mathrm{~km} / \mathrm{h}$ is strongly dependent on the wheelbase. A $40 \%$ decreased wheelbase leads to a $27 \%$ decreased turning radius at $10 \mathrm{~km} / \mathrm{h}$. For velocities above $\sim 27 \mathrm{~km} / \mathrm{h}$, the wheelbase has no significant effect on the turning radius. This is because the tire side forces have reached their maximum values and further increasing slip angles do not increase the side forces. The yaw velocity gain plots show that the characteristic speed is smaller for shorter wheelbases. This can also be seen in the turning radius plot in which the graph bends at lower velocities for smaller wheelbases. Therefore, a vehicle with a smaller wheelbase starts to under-steer at a smaller velocity for certain steering angles, but the turning radius is the same for higher velocities. 

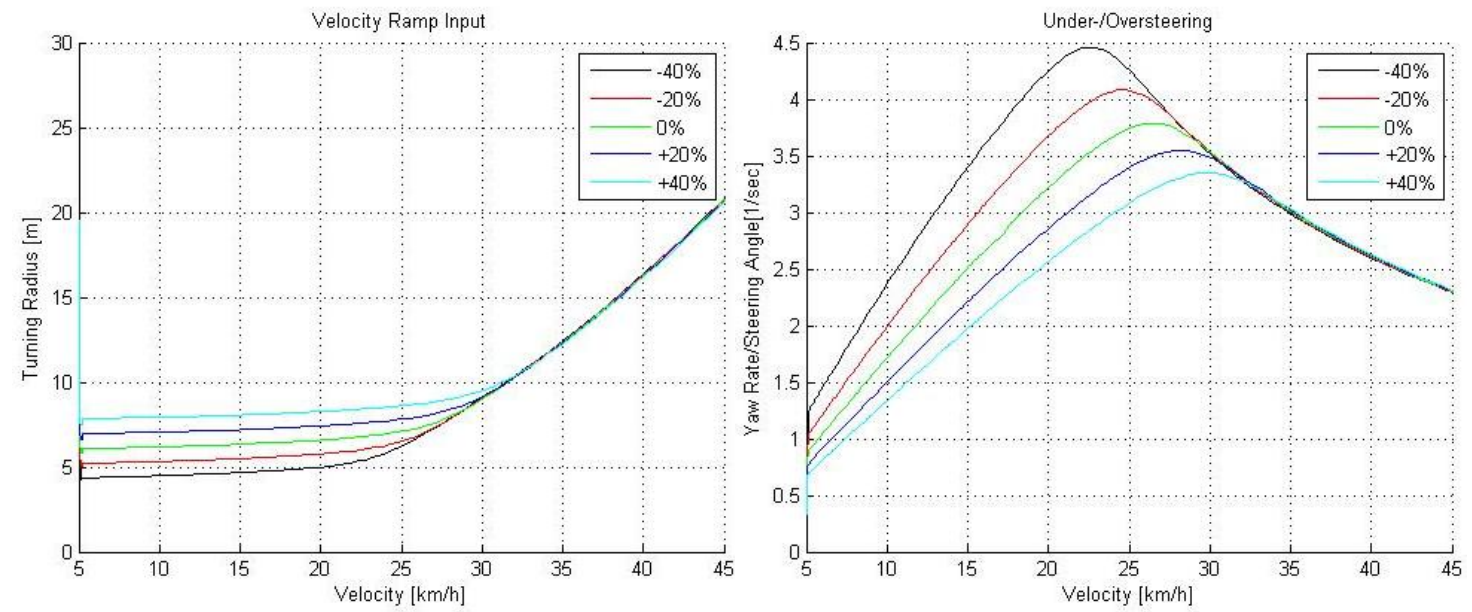

Figure 13. Turning radius versus velocity (left) and yaw velocity gain (right) for an understeering vehicle with different wheelbases (percent change) at a steering angle of 15 degrees.

Front and Rear Tire Combination

The under- and over-steering behavior can also be influenced by choosing different tires for the front wheels than for the rear wheels. To prevent over-steering, the rear tires must be able to carry more lateral forces and the front tires less. In order to show this effect, a particular vehicle is analyzed with front tires that have a $10 \%$ smaller coefficient of friction compared to the rear tires. Figure 14 shows the original over-steering vehicle on the left and the same vehicle with different front tires on the right. It can be seen that the vehicle with the different front tires is not over-steering, and instead shows under-steering behavior. The same principle can be used to avoid or minimize under-steering. Hence, choosing a different tire combination affects the vehicle behavior, but can also increase the turning radius poor tire selection is employed either in the front or in the rear.
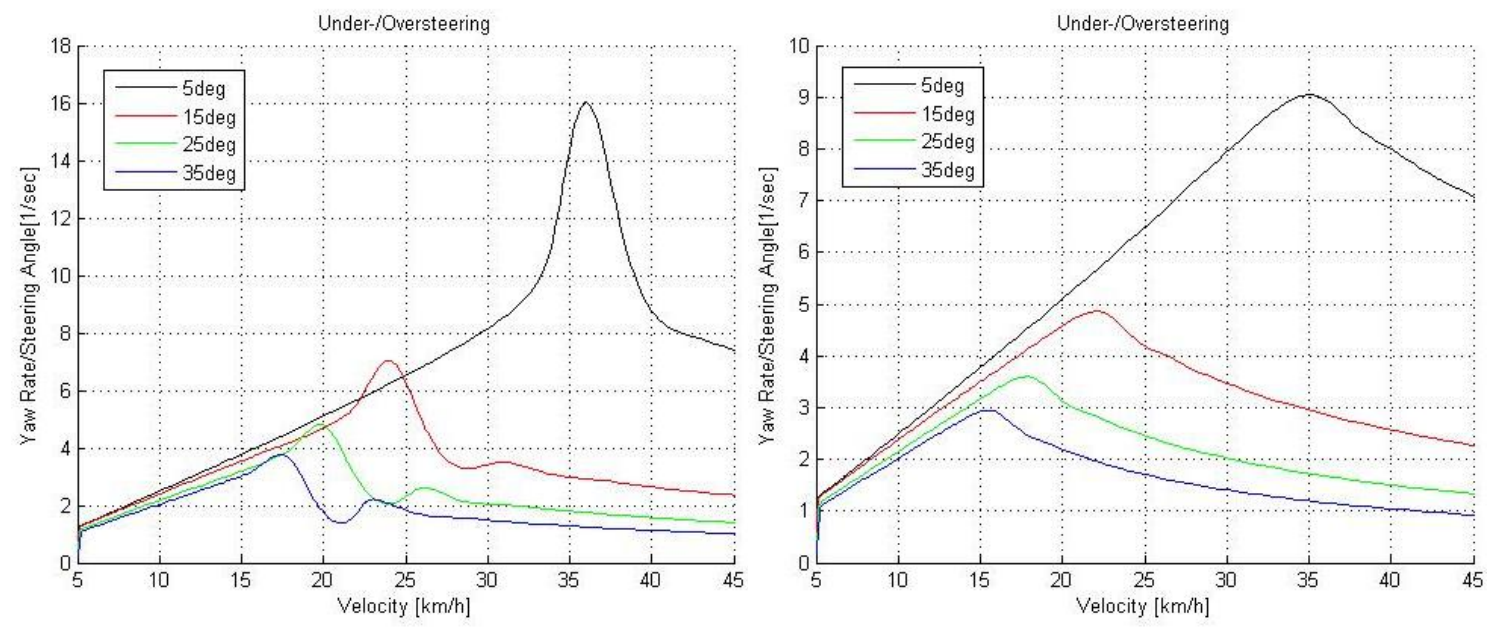

Figure 14. Over-steering vehicle on the left, on the right with a 0.1 smaller coefficient of friction on the front tires 
Tutorial

This section describes, in brief, how future students might set up the program and perform simulations in order to evaluate a design. It begins with describing which system-variables are required for the input of the simulation tool. The tutorial also shows how to implement these data into the dynamic simulation tool and how to setup the driver and road inputs. Furthermore it briefly describes how implemented loops can be used for comfortable parameter variation analyses. Additionally, it shows how to obtain the resulting graphs and in which way the data can be saved.

\section{System Variable Initialization}

The tool is capable of using any consistent set of units. Table 1 is an example of the parameter set up which shows the required system-variables and their line number in the appropriate MATLAB file.

Table 1: System-variables saved in the "VehicleParameter.m" file

\begin{tabular}{|r|l|c|c|c|}
\hline$\#$ & \multicolumn{1}{|c|}{ System-Variable } & Symbol in M-File & SI Unit & Line No. \\
\hline 1 & Wheel base & $\mathrm{L}$ & $\mathrm{m}$ & 7 \\
\hline 2 & Track width front & $\mathrm{wf}$ & $\mathrm{m}$ & 8 \\
\hline 3 & Track width rear & $\mathrm{wr}$ & $\mathrm{m}$ & 9 \\
\hline 4 & Ratio front axle to COG over wheelbase & $\mathrm{aoL}$ & - & 10 \\
\hline 5 & Roll center height front & $\mathrm{hrcf}$ & $\mathrm{m}$ & 14 \\
\hline 6 & Roll center height rear & $\mathrm{hrcr}$ & $\mathrm{m}$ & 15 \\
\hline 7 & Vertical COG position & $\mathrm{hwc}$ & $\mathrm{m}$ & 16 \\
\hline 8 & Unsprung mass front & $\mathrm{mwr}$ & $\mathrm{kg}$ & 19 \\
\hline 9 & Unsprung mass rear & $\mathrm{mcar}$ & $\mathrm{kg}$ & 20 \\
\hline 10 & Sprung mass (incl. driver + fuel) & $\mathrm{Ix}$ & $\mathrm{kg} \mathrm{m}^{2}$ & 21 \\
\hline 11 & MMOI about roll axis & $\mathrm{ly}$ & $\mathrm{kg} \mathrm{m}^{2}$ & 25 \\
\hline 12 & MMOI about pitch axis & $\mathrm{Iz}$ & $\mathrm{kg} \mathrm{m}^{2}$ & 26 \\
\hline 13 & MMOI about yaw axis & $\mathrm{kwf}$ & $\mathrm{N} / \mathrm{m}$ & 29 \\
\hline 14 & Tire spring stiffness front & $\mathrm{kwr}$ & $\mathrm{N} / \mathrm{m}$ & 30 \\
\hline 15 & Tire spring stiffness rear & $\mathrm{cwf}$ & $\mathrm{Ns} / \mathrm{m}$ & 31 \\
\hline 16 & Tire damping coeff. Front & $\mathrm{cwr}$ & $\mathrm{Ns} / \mathrm{m}$ & 32 \\
\hline 17 & Tire damping coeff. rear & $\mathrm{Rwf}$ & $\mathrm{m}$ & 35 \\
\hline 18 & Tire radius front & $\mathrm{Rwr}$ & $\mathrm{m}$ & 36 \\
\hline 19 & Tire radius rear & $\mathrm{Iwf}$ & $\mathrm{kg} \mathrm{m}^{2}$ & 37 \\
\hline 20 & Tire MMOI front & $\mathrm{Iwr}$ & $\mathrm{kg} \mathrm{m}^{2}$ & 38 \\
\hline 21 & Tire MMOI rear & $\mathrm{krf}$ & $\mathrm{N} / \mathrm{m}$ & 41 \\
\hline 22 & Spring stiffness anti-roll bar front & $\mathrm{krr}$ & $\mathrm{N} / \mathrm{m}$ & 42 \\
\hline 23 & Spring stiffness anti-roll bar rear & $\mathrm{Sarm}$ & $\mathrm{m}$ & 46 \\
\hline 24 & Steering arm length & $\mathrm{AA}$ & $\mathrm{deg}$ & 47 \\
\hline 25 & Ackermann Angle & $\mathrm{kcf}$ & $\mathrm{N} / \mathrm{m}$ & 71 \\
\hline 26 & Suspension spring stiffness front & $\mathrm{N} / \mathrm{m}$ & 72 \\
\hline 27 & Suspension spring stiffness rear & $\mathrm{Ns} / \mathrm{m}$ & 73 \\
\hline 28 & Suspension damping coeff. font & $\mathrm{Ns} / \mathrm{m}$ & 74 \\
\hline 29 & Suspension damping coeff. rear & & \\
\hline & & & & \\
\hline
\end{tabular}

The tire coefficients are not included in this table, but students can switch between on- and offroad tires by activating the corresponding shape function parameter in the Simulink subsystem and by changing the surface friction coefficient. Note that the steering system may be analyzed by changing any or all of the values, e.g., the Ackermann Angle (parameter \#25). A positive 
value represents the Ackermann steering geometry, zero indicates parallel steering, and a negative value refers to reverse Ackermann steering geometry.

Driver and road inputs may be created and stored as data files or time series vectors to simulate maneuvers in certain conditions. All driver and road inputs are implemented in a "Control.m" file, the master $m$-file, which calls all other $\mathrm{m}$-files and subsequently runs the appropriate subsystem simulation programs.

\section{Conclusion}

A vehicle dynamics design tool and tutorial has been completed by students at the Milwaukee School of Engineering. The tool is valuable for senior design students considering parameter decisions early in the process of vehicle design. Suspension, cornering, roll-over, and handling aspects are investigated. MATLAB and Simulink files are open-source documents allowing full customization by future students.

\section{Acknowledgements}

The work of students Jonah Splettstoesser and Armin Haugg are greatly appreciated and form the bulk of the results in this work.

\section{References}

[1] Splettstoessr, Jonah M. Developing a Simulation Tool for Vehicle Dynamics and Rollover of the Baja Buggy and Formula Hybrid Car. Milwaukee School of Engineering, Fachhochschule Luebeck, 2010. Diplomarbeit.

[2] Haugg, Armin. Analysis and Simulation of the Dynamic Steering Response for an SAE Baja-Car. Milwaukee School of Engineering, Fachhochschule Luebeck, 2008. Diplomarbeit.

[3] Gillespie, Thomas D. Fundamentals of Vehicle Dynamics. Warrendale : Society of Automotive Engineers, Inc., 1992. ISBN 1-56091-199-9.

[4] Heiszing, Bernd and Ersoy, Metin. Fahrwerkhandbuch. Wiesbaden : Vieweg \& Sohn Verlag | GWV Fachverlage GmbH, 2007. ISBN 978-3-8348-0105-0.

[5] Euler angles. Wikipedia, the free encyclopedia. [Online] [Cited: January 19, 2010.] http://en.wikipedia.org/wiki/Euler_angles.

[6] Gimbal Lock. Wikipedia, the free encyclopedia. [Online] [Cited: January 24, 2010.] http://en.wikipedia.org/wiki/Gimbal_lock.

[7] D. C. Holloway, Examination of ATV Tire Forces Generated on Clay, Grass, and Sand Surfaces, SAE Paper 891106, 1989. 